\title{
BMJ Open Rates of retention of persons with a mental health disorder in outpatient smoking cessation and reduction trials, and associated factors: protocol for a systematic review and meta-analysis
}

\author{
Alexandra Patricia Metse, ${ }^{01,2}$ Emily Stockings, ${ }^{3}$ Jacqueline Bailey, ${ }^{1}$ \\ Timothy Regan, ${ }^{1}$ Kate Bartlem, ${ }^{1}$ Luke Wolfenden, ${ }^{4,5}$ Gemma Taylor, ${ }^{6}$ \\ John Wiggers, ${ }^{4,5}$ Jenny Bowman ${ }^{1}$
}

To cite: Metse AP, Stockings E, Bailey J, et al. Rates of retention of persons with a mental health disorder in outpatient smoking cessation and reduction trials, and associated factors: protocol for a systematic review and meta-analysis. BMJ Open 2019;9:e030646. doi:10.1136/ bmjopen-2019-030646

- Prepublication history and additional material for this paper are available online. To view please visit the journal (http:// dx.doi.org/10.1136/bmjopen2019-030646)

Received 26 March 2019 Revised 20 June 2019 Accepted 31 July 2019
D) Check for updates

(c) Author(s) (or their employer(s)) 2019. Re-use permitted under CC BY-NC. No commercial re-use. See rights and permissions. Published by BMJ.

For numbered affiliations see end of article.

\section{Correspondence to} Dr Alexandra Patricia Metse; alexandra.metse@murdoch. edu.au

\section{ABSTRACT}

Introduction Smoking among persons with a mental health disorder is associated with inequitable health, social and economic burden. Randomised controlled trials (RCTs) are considered the gold standard design for the assessment of healthcare intervention efficacy/ effectiveness. However, many RCTs of smoking interventions for persons with a mental health disorder lack rigour due to low participant retention. No systematic review has pooled retention rates in randomised trials of smoking interventions for persons with a mental health disorder or explored associated factors. The aims of the systematic review will therefore be to: (1) summarise overall rates of participant retention in smoking cessation and reduction trials involving persons with a mental health disorder (including for experimental and control groups separately) and (2) determine if retention rates vary according to participant, environmental, researcher and study factors.

Methods and analysis PsycINF0, EMBASE, MEDLINE, CENTRAL and The Cochrane Tobacco Addiction Review Group Specialised Register will be searched for reports of RCTs of outpatient smoking cessation or reduction interventions for adults with a mental health disorder. The search terms will include MeSH terms and free text words, and there will be no language or date restrictions. All databases will be searched from inception to present. Data will be analysed using the Mantel-Haenszel fixedeffect model, and where substantial heterogeneity $\left(l^{2}\right.$ $>50 \%$ ) is detected, DerSimonian \& Laird inverse-variance random effects model. Pooled estimates and $95 \% \mathrm{Cls}$ will be calculated for overall participant retention rates and for intervention and control trial arms separately. Associations between participant retention and participant, environmental, researcher and study factors will be assessed via subgroup analyses and, where sufficient data are obtained, meta-regression.

Ethics and dissemination This study does not require ethical approval. The findings of this review will be disseminated via publication in a peer-reviewed open access medical journal and presentations at international scientific meetings.
Strengths and limitations of this study

- This will be the first systematic review to pool retention rates in randomised controlled trials of outpatient smoking interventions for persons with a mental health disorder and explore associated factors.

- Identification of participant, environmental, researcher and study factors associated with retention will be informative when designing future smoking intervention trials for persons with a mental health disorder and may lead to increased retention and, in turn, rigour of research in the field.

- It will employ Cochrane's methods for the conduct of systematic reviews.

- Trials of smoking interventions delivered exclusively in psychiatric inpatient setting will not be included.

\section{INTRODUCTION}

Tobacco smoking is a leading cause of preventable morbidity and mortality worldwide. ${ }^{1}$ Smoking prevalence has steadily declined to between $13 \%$ and $20 \%$ over the past 40 years in the general population of high-income countries; ${ }^{2-4}$ however, it has remained unchanged among persons with a mental health disorder. ${ }^{56}$ Population surveys suggest that $40 \%, 44 \%$ and $36 \%$ of community residing persons with a mental health disorder in the USA, ${ }^{7} \mathrm{UK}^{8}$ and Australia ${ }^{7}$ smoke, respectively, with prevalence estimates shown to increase as mental illness severity and number of life time disorders increase. ${ }^{9} 10$ These groups are also reported to smoke more heavily and be more nicotine dependent than smokers without a mental health disorder ${ }^{9-11}$ and have been estimated to consume up to $45 \%$ of all cigarettes sold in high-income countries. ${ }^{912} 13$ Smoking among 
persons with a mental health disorder is consequently associated with inequitable health, social and economic burden. ${ }^{14}$ Despite this, many high-quality randomised trials of smoking interventions exclude persons with a mental health disorder ${ }^{1516}$ and those focused on this population group often lack rigour due to, for example, small sample size and low participant retention. ${ }^{17-19}$ More rigorous intervention research is needed to address this international public health issue. ${ }^{2021}$

Randomised controlled trials (RCTs) are considered the gold standard design for assessment of healthcare intervention efficacy and effectiveness. ${ }^{22}$ In any trial, low rates of participant retention for follow-up data collection can compromise the internal and external validity and reduce statistical power. ${ }^{23-25}$ Threats to internal validity are particularly salient as retention decreases $\left(<80 \%{ }^{25}\right)$ or is differential between allocation groups,${ }^{26}$ increasing the risk of bias and confounding. ${ }^{26}$ Additionally, statistical methods (eg, multiple imputation) cannot account entirely for the impact of low/differential retention and have been noted to occasionally be used inappropriately. ${ }^{27}$ Illustrating a consensus regarding the need to consider and address the issue of low retention in clinical and health behaviour trials: the inclusion of items on participant retention in the Consolidated Standards of Reporting Trials (CONSORT) statement, ${ }^{28}{ }^{29}$ and prioritisation of trial methodology research to develop novel approaches to increase retention in randomised trials. ${ }^{3031}$ In addition, the emergence of recent systematic reviews examining retention rates in health behaviour studies ${ }^{32-36}$ and publication of retention data from high quality randomised trials ${ }^{37-39}$ indicates increased interest in the topic of retention and a recognition of its importance.

Participant retention is problematic in trials of smoking cessation interventions, ${ }^{40} 41$ including those involving persons with a mental health disorder. ${ }^{17-19}$ For example, review of risk of bias assessments in systematic reviews of smoking cessation intervention trials involving persons with depression ${ }^{17}$ and substance use disorders ${ }^{19}$ revealed retention rates as low as $27 \%-33 \%$, at trial end points. In addition, 65 of the trials $\left(34 / 49 ;{ }^{17} 31 / 35^{19}\right)$ included in the reviews transparently reported on participant retention, of which $40\left(22 / 34 ; ;^{17} 18 / 31^{19}\right)$ achieved retention rates less than $80 \%$. In addition, there was evidence of differential retention ( $\geq 10 \%$ difference in retention rates between allocation groups) in $13\left(5 / 34 ;{ }^{17} 8 / 31^{19}\right)$ trials, with this figure likely an underestimate due to non-systematic reporting of retention data according to allocation group. No study has systematically and quantitatively summarised retention rates of persons with a mental health disorder in smoking trials or considered factors that may impact on such rates.

Factors likely to impact participant retention in any trial could be categorised into four types: (1) 'participant' (eg, demographic information) (2) 'environmental' (eg, recruitment method/setting) (3) 'researcher' (eg, staff qualifications) and (4) 'study' (eg, trial design).$^{42}$ In terms of participant factors, evidence from single studies in the field of smoking and mental illness suggests younger age, higher income, less severe mental health symptomology and readiness to quit at baseline may be associated with higher retention in trials. ${ }^{37} 394344$ While no research has reported on environmental and researcher factors associated with retention in smoking trials involving persons with a mental health disorder, findings from the broader field of smoking research suggest that higher retention rates are observed when proactive (compared with reactive) recruitment methods are adopted, ${ }^{40}$ and staff delivering the intervention receive more comprehensive training. ${ }^{29}$ With regard to study factors, Cochrane systematic review evidence suggests open-label designs increase the relative risk of higher retention rates (risk ratio (RR) $1.37 ; 95 \%$ CI 1.16 to 1.63 ) in randomised trials of health interventions outside the field of smoking. ${ }^{33}$ Further, adoption of strategies with demonstrated effectiveness in improving retention in health research could also be considered a 'study' factor. Such strategies were developed based on identified barriers to participant retention $^{32}$ and include monetary reimbursement for time, ${ }^{45}$ provision of reminders for follow-up assessments ${ }^{46}$ and participant tracking systems. ${ }^{46}$ Identification of participant, environmental, researcher and study factors associated with higher participant retention in smoking trials involving persons with a mental health disorder will be informative for researchers when designing definitive trials and in turn potentially improve the rigour of available intervention research in the field.

No review has systematically examined rates of participant retention or explored associated factors in smoking cessation and/or reduction trials involving persons with a mental health disorder. Therefore, the aims of the proposed systematic review will be to: (1) summarise overall rates of participant retention in such trials (including for experimental and control groups separately) and (2) determine if retention rates vary according to participant, environmental, researcher and study factors.

\section{METHODS}

This protocol adheres to the preferred reporting items for systematic review and meta-analysis protocols (PRISMA-P) statement. ${ }^{47}$

\section{Inclusion criteria}

Studies identified by a Cochrane review of outpatient smoking cessation and reduction interventions for persons with a mental health disorder ${ }^{48}$ will be included. Criteria for inclusion:

- Study design: RCTs and cluster RCTs.

- Participants: adult (aged 18 years or above) smokers, who have either been diagnosed with a mental health disorder (determined via medical record or self-report) in the past 12 months or are currently receiving treatment for the same. Participants will not be required to express an intention to 
quit smoking to be eligible. No limits will be placed on recruitment setting; however, the majority of the intervention component must be delivered in a community setting. Interventions delivered exclusively during an inpatient psychiatric admission will be excluded as they are the focus of another Cochrane review. ${ }^{49}$

- Intervention type: any intervention to aid smoking cessation or reduction, for example, pharmacotherapy, psychoeducation, cognitive and behavioural therapies. Interventions aiming to increase the uptake/utilisation of smoking cessation supports will be considered. However, whole-of-setting interventions (eg, smoke-free policy) will be excluded.

- Intervention delivery: face-to-face, telephone, online, mail, individual and/or group. Interventions can be facilitated by any person (eg, research officer or healthcare clinician) or via unmoderated online/ phone-based methods.

- Control: all control conditions will be considered, for example, placebo, no treatment, usual care, other smoking cessation/reduction interventions.

- Follow-up: undertaken at least 6 months postbaseline assessment. $^{50}$

\section{Search strategy}

The following databases will be searched for reports of trials of smoking cessation and reduction interventions among smokers with a mental health disorder:

- The Cochrane Tobacco Addiction Review Group Specialised Register.

- Cochrane Central Register of Controlled Trials (CENTRAL).

- MEDLINE (OVID SP 1946 to present) \& MEDLINE in-process \& other non-indexed citations (OVID SP).

- EMBASE (OVID SP, 1947 to present).

- PsycINFO (OVID SP,1806 to present).

Search terms will include MeSH terms and free text words, and there will be no language or date restrictions. All databases will be searched from inception to present. Reference lists of included studies will be checked for other relevant research. Online supplementary appendix 1 comprises the proposed Medline search strategy. Searches will likely be undertaken in December 2019.

In addition to the above, we will search international clinical trials registers for recently completed trials, including: the Australian and New Zealand Clinical Trials Registry (ANZCTR), UK Clinical Trials Gateway; US Clinical Trials Register and the WHO Portal.

\section{Study screening and data extraction}

Two review authors will independently screen the titles and abstracts of search results for relevance, acquire and screen the full texts of potentially eligible articles and extract data from included studies. Disagreements during the screening and data extraction processes will be resolved by referral to a third review author. Covidence (https://www.covidence.org/home) and Microsoft
Excel will be used to manage data during screening and extraction phases.

Data to be extracted from included trials are outlined in table 1, with independent variables for consideration identified in the respective column and summarised under relevant headings below. A large number of potential independent variables could be considered, selection will be guided by extant literature, experts in the field and, with exception of 'participant factors', focus on factors modifiable by researchers.

\section{Participant factors}

Participant characteristics for consideration, collected at the baseline assessment of individual studies, may include: age ${ }^{39}{ }^{40}$ gender, ${ }^{4043}$ race/ethnicity, ${ }^{40}$ socioeconomic status, ${ }^{40}$ mental health diagnosis,${ }^{37}$ severity of distress,${ }^{13}$ motivation/readiness to quit smoking, ${ }^{40} 44$ nicotine dependence, ${ }^{3040}$ daily cigarette consumption ${ }^{40} 49$ and requirement to set a quit date on recruitment. ${ }^{40}$

\section{Environmental factors}

Environmental factors comprise those related to the study setting. Such factors that may be considered include the method $^{40}$ and setting ${ }^{51}$ of recruitment and outcome data collection.

\section{Researcher factors}

Researcher factors include characteristics, skills and roles of the staff that design and implement the study and may include: recruiter qualifications, training and professional role $^{5152}$ and relationship of recruiter to participants. ${ }^{51}$

\section{Study factors}

Study factors comprise features of the study design and implications of such for participants. A number of study factors may be considered: blinding/masking of research personnel, ${ }^{33}$ adoption of retention strategies, ${ }^{32} 37$ timing of follow-up assessments, ${ }^{39} 40$ biochemical validation of self-reported abstinence and potential cost/s to participants of follow-up assessment completion. ${ }^{32} 51$

\section{Outcomes}

The primary outcome of interest for the review is participant retention rate at the longest follow-up. Within each study, retention will be defined as participation in the final follow-up assessment of a trial (eg, completion of telephone or face-to-face interview or response to postal and electronic questionnaires). Retention rate will be calculated using the formula: number retained/number recruited. Study authors will be approached to obtain data required to calculate outcome measures, if not reported in the published manuscript. Secondary analyses will explore follow-up rates separately at other time points, for example, at 6 months, 12 months and 18 months.

\section{Analysis}

Data will be analysed using Review Manager 5.3 and Stata 15.0. Outcome data will be collected in accordance with intention-to-treat principles, where all recruited 
Table 1 Data extraction fields

Considered

Author

First author last name

Year

Title

Country

Years of study

Funding source

Conflict of interests

\section{Methods}

Study design

Aims of study

Years of recruitment

Method of recruitment

\section{Recruitment setting}

Recruiter qualifications, training and professional role

Familiarity of recruiter to participant

Retention strategy employed

\section{Participants}

Age

Gender

Race/ethnicity

Socio-economic status

Mental health diagnosis

Severity of distress/ mental health disorder

Inclusion/exclusion criteria

Current smoker definition

Cigarette consumption (baseline)

Nicotine dependence (baseline)

Motivation to quit (baseline)

Yes/no; type

From text or similar measures

From text measures
Year published

Title of publication

From text

Year/s study was conducted

From text

From text

\section{RCT, cluster RCT}

From text

Years

Face-to-face, social media, random-digit dialling;; snowball recruiting; use of social networks; advertisements, media or notices ; identification of potential participants through public registries, medication record and so on

Hospital, community healthcare, primary care, non-government Yes organisation (NGO), other staff member in hospital), regular clinician, peer, other

Mean (standard deviation), median (range)

Male, female, other/unspecified.

Yes Yes

Employment status, level of education or similar

Yes

Type of mental illness/es of participants

Yes

Daily, weekly, occasional smoker

Yes

Mean (standard deviation), median (range)

Yes

Fagerstrom Test of Nicotine Dependence (FTND) or similar $\quad$ Yes

Readiness and Motivation to Quit Smoking Questionnaire or $\quad$ Yes similar measures

Requirement to set a quit date on recruitment Yes/no Yes

\section{Intervention}

Treatment type

Pharmacotherapy, psychoeducation, cognitive and behavioural therapies, staff training, other

Dosage (if applicable)

Duration Weeks

Number of session (where applicable)

Facilitator qualifications and training From text 


\section{Outcomes}

\begin{tabular}{|c|c|c|}
\hline Primary outcomes & Specified and collected & \\
\hline Secondary outcomes & Specified and collected & \\
\hline Definition of abstinence & From text & \\
\hline Definition of smoking reduction & From text & \\
\hline Type of biochemical validation (if applicable) & Carbon monoxide (CO), cotinine, other & Yes \\
\hline Timing of follow-up assessments & Months & Yes \\
\hline Mode of follow-up assessment & $\begin{array}{l}\text { Face-to-face, telephone interview, postal or electronic survey } \\
\text { and so on }\end{array}$ & Yes \\
\hline Follow-up setting (if applicable) & & Yes \\
\hline $\begin{array}{l}\text { Potential costs to participant associated with } \\
\text { follow-up assessments }\end{array}$ & Absence from work, fuel and parking costs and so on & Yes \\
\hline $\begin{array}{l}\text { Intention to treat and per protocol analyses } \\
\text { conducted }\end{array}$ & Yes/no & \\
\hline \multicolumn{3}{|l|}{ Risk of bias } \\
\hline \multicolumn{3}{|l|}{ Method of randomised sequence generation } \\
\hline \multicolumn{3}{|l|}{ Method of allocation concealment } \\
\hline Blinding of participants & Yes/no & Yes \\
\hline Blinding of research staff & Yes/no & Yes \\
\hline Blinding of outcome assessors & Yes/no & Yes \\
\hline \multicolumn{3}{|l|}{ Levels of loss to follow-up } \\
\hline \multicolumn{3}{|l|}{ Reporting of loss to follow-up } \\
\hline GRADE & GRADE methodology rating & \\
\hline
\end{tabular}

Placebo, no treatment, waitlist, usual care, other smoking cessation/reduction intervention, other

participants will be included in denominator figure of the retention algorithm. Data that are missing due to participant death will be deducted from the total sample size for all analyses.

\section{According to study aim}

Aim 1: the proportion of participants retained will be pooled using the Stata 15.0 prevalence command, 'metaprop' using the Freeman-Tukey double arcsine transformation to stabilise variances and prevent exclusion of studies where proportions approached 0 or $1 .^{53}{ }^{54} \mathrm{We}$ will calculate pooled estimates and $95 \%$ CIs for overall participant retention rates as well as for intervention and control trial arms separately. Differences between intervention and control arms will be assessed via risk ratios. Data will be analysed using the Mantel-Haenszel fixed-effect model. If substantial heterogeneity $\left(\mathrm{I}^{2}>50 \%\right)^{50}$ is detected in the pooled estimates, we will run sensitivity analyses using DerSimonian \& Laird inverse-variance random effects meta-analysis. ${ }^{55}$
Aim 2: the association between participant, environmental, researcher and study factors and overall participant retention will be assessed via subgroup analyses and, where sufficient data are obtained ( $>10$ observations) ${ }^{50}$ meta-regression. Subgroup analyses will be undertaken in line with the meta-analysis approach described for Aim 1. Independent variables (table 1) will be categorised and reduced to two or three levels for the purpose of this analysis. In terms of the meta-regression, we will build models using the 'regress' and, where relevant, 'metareg' commands. Study level weights will be obtained from the fixed effects meta-analysis and will be included in the regression models using the analytical weights option. Categorical and numerical independent variables to be considered in these models. Ratio data will be natural $\log$ transformed prior to analyses. Where data are not amenable to meta-analysis, factors reported in individual studies to be associated with retention will be summarised via narrative synthesis. 
Statistical heterogeneity and sensitivity analyses

The $I^{2}$ statistic, which describes the percentage of total variation across studies that is due to heterogeneity rather than chance, will be used to assess statistical heterogeneity of pooled data. Heterogeneity in pooled estimates will be described as low $\left(I^{2} \sim 25 \%\right)$, moderate $\left(I^{2} \sim 50 \%\right)$ or high $\left(I^{2} \sim 75 \%\right) .{ }^{56}$ Sensitivity analyses will be conducted to explore the impact of including studies with an overall high risk of bias.

\section{Patient and public involvement}

Patients/public were not involved at any stage in the development of this protocol.

\section{DISCUSSION}

This rigorous systematic review will be the first to pool retention rates in RCTs of outpatient smoking interventions for persons with a mental health disorder. Identification of participant, environmental, researcher and study factors associated with retention will be informative when designing future smoking intervention trials for persons with a mental health disorder, and in turn, may lead to increased rigour of research in the field due to higher participant retention. Findings may not generalise to trials of smoking interventions delivered exclusively in psychiatric inpatient settings.

\section{Author affiliations}

${ }^{1}$ School of Psychology, University of Newcastle, Callaghan, New South Wales, Australia

${ }^{2}$ Discipline of Psychology, Murdoch University, Murdoch, Western Australia, Australia ${ }^{3}$ National Drug and Alcohol Research Centre, University of New South Wales, Sydney, New South Wales, Australia

${ }^{4}$ School of Medicine and Public Health, University of Newcastle, Callaghan, New South Wales, Australia

${ }^{5}$ Hunter New England Population Health, Wallsend, New South Wales, Australia

${ }^{6}$ Department of Psychology, University of Bath, Bath, BA2 7AY, United Kingdom

Acknowledgements The authors would like to acknowledge all contributors to the overarching Cochrane systematic review.

Contributors APM, ES, JB and JW conceived the review. ES, JB and JW designed the review, and $A M$ is the guarantor and led the writing of the protocol. TR, JB, $\mathrm{KB}, \mathrm{GT}$ and $\mathrm{LW}$ provided critical input across all stages. The corresponding author attests that all listed authors meet authorship criteria and that no others meeting the criteria have been omitted.

Funding ES and KB are supported by Australian National Health and Medical Research Council Early Career Fellowships (ES \#APP1104600; KB \#APP1142272). GT is funded by Cancer Research UK Population Researcher Postdoctoral Fellowship award (reference: C56067/A21330).

Disclaimer Funders did not contribute, at any stage, to the development of this protocol or decision to publish.

Competing interests None declared.

Patient consent for publication Not required.

Provenance and peer review Not commissioned; externally peer reviewed.

Open access This is an open access article distributed in accordance with the Creative Commons Attribution Non Commercial (CC BY-NC 4.0) license, which permits others to distribute, remix, adapt, build upon this work non-commercially, and license their derivative works on different terms, provided the original work is properly cited, appropriate credit is given, any changes made indicated, and the use is non-commercial. See: http://creativecommons.org/licenses/by-nc/4.0/.

\section{REFERENCES}

1. Lim SS, Vos T, Flaxman AD, et al. A comparative risk assessment of burden of disease and injury attributable to 67 risk factors and risk factor clusters in 21 regions, 1990-2010: a systematic analysis for the global burden of disease study 2010. The Lancet 2012;380:2224-60.

2. Australian Institute of Health and Welfare (AIHW). National drug strategy household survey detailed report 2013. drug statistics series No. 28. cat. No. Phe 183. Canberra: AlHW, 2014.

3. Centers for Disease Control and Prevention. Current cigarette smoking among adults-United states, 2005-2014. Morb Mortal Wkly Rep 2015;64:1233-40.

4. Ministry of Health. Annual update of key results 2014/15: new Zealand health survey. Wellington: Ministry of Health, 2015.

5. The Lancet. Smoke alarm: mental illness and tobacco. The Lancet 2013;381.

6. Szatkowski L, McNeill A. Diverging trends in smoking behaviors according to mental health status. Nicotine Tob Res 2015;17:356-60.

7. Lawrence D, Mitrou F, Zubrick SR. Smoking and mental illness: results from population surveys in Australia and the United States. BMC Public Health 2009;9:285.

8. Farrell M, Howes S, Bebbington P, et al. Nicotine, alcohol and drug dependence and psychiatric comorbidity. Br J Psychiatry 2001;179:432-7.

9. Lasser K, Boyd W, Woolhandler S, et al. Smoking and mental illness: a population based study. JAMA 2000;284:606-10.

10. de Leon J, Diaz FJ. A meta-analysis of worldwide studies demonstrates an association between schizophrenia and tobacco smoking behaviors. Schizophr Res 2005;76:135-57.

11. Bowden JA, Miller CL, Hiller JE. Smoking and mental illness: a population study in South Australia. Aust N Z J Psychiatry 2011;45:325-31.

12. Grant BF, Hasin DS, Chou SP, et al. Nicotine dependence and psychiatric disorders in the United States: results from the Nationa epidemiologic survey on alcohol and related conditions. Arch Gen Psychiatry 2004;61:1107-15.

13. McManus S, Meltzer H, Campion J. Cigarette smoking and mental health in England: data from the adult psychiatric morbidity survey 2007. London: National Centre for Social Research, 2010.

14. Colton CW, Manderscheid RW. Congruencies in increased mortality rates, years of potential life lost, and causes of death among public mental health clients in eight states. Prev Chronic Dis 2006;3:A42.

15. Schroeder SA, Morris CD. Confronting a Neglected Epidemic: Tobacco Cessation for Persons with Mental Illnesses and Substance Abuse Problems. In: Fielding JE, Brownson RC, Green LW, eds. Annual review of public health, vol 31. annual review of public health. 31. Palo Alto: Annual Reviews, 2010: 31. 297-314.

16. Evins AE. Reassessing the safety of varenicline. AJP 2013;170:1385-7.

17. van der Meer RM, Willemsen MC, Smit F, et al. Smoking cessation interventions for smokers with current or past depression. Cochrane Database Syst Rev 2013;38.

18. Tsoi DT, Porwal M, Webster AC, et al. Interventions for smoking cessation and reduction in individuals with schizophrenia. Cochrane Database Syst Rev 2013;1.

19. Apollonio D, Philipps R, Bero L, et al. Interventions for tobacco use cessation in people in treatment for or recovery from substance use disorders. Cochrane Database Syst Rev 2016;105.

20. Metse AP, Wiggers JH, Wye PM, et al. Smoking and mental illness: a bibliometric analysis of research output over time. NICTOB 2017;19:24-31.

21. Royal College of Physicians, Royal College of Psychiatrists. Smoking and mental health. London: RCP Council Report CR1782013.

22. Spieth PM, Kubasch AS, Penzlin Al, et al. Randomized controlled trials - a matter of design. Neuropsychiatr Dis Treat 2016;12:1341-9.

23. Dettori J, follow-up Lto. Loss to follow-up. Evid Based Spine Care $J$ 2011;2:7-10.

24. Schulz KF, Grimes DA. Sample size slippages in randomised trials: exclusions and the lost and wayward. The Lancet 2002;359:781-5.

25. Dumville JC, Torgerson DJ, Hewitt CE. Reporting attrition in randomised controlled trials. BMJ 2006;332:969-71.

26. Fewtrell MS, Kennedy K, Singhal A, et al. How much loss to followup is acceptable in long-term randomised trials and prospective studies? Arch Dis Child 2008;93:458-61.

27. Molnar FJ, Hutton B, Fergusson D. Does analysis using "last observation carried forward" introduce bias in dementia research? Can Med Assoc J 2008;179:751-3.

28. Moher D, Hopewell S, Schulz KF, et al. Consort 2010 explanation and elaboration: updated guidelines for reporting parallel group randomised trials. J Clin Epidemiol 2010;63:e1-37. 
29. Moher D, Schulz KF, Altman DG. The CONSORT statement: revised recommendations for improving the quality of reports of parallelgroup randomised trials. The Lancet 2001;357:1191-4.

30. Tudur Smith C, Hickey H, Clarke M, et al. The trials methodological research agenda: results from a priority setting exercise. Trials 2014;15:32.

31. Treweek S, Bevan S, Bower P, et al. Trial forge guidance 1: what is a study within a trial (SWAT)? Trials 2018;19:139.

32. Bonevski B, Randell M, Paul C, et al. Reaching the hard-to-reach: a systematic review of strategies for improving health and medical research with socially disadvantaged groups. BMC Med Res Methodol 2014;14:1-29.

33. Brueton VC, Tierney JF, Stenning S, et al. Strategies to improve retention in randomised trials: a Cochrane systematic review and meta-analysis. BMJ Open 2014;4:e003821.

34. Booker CL, Harding S, Benzeval M. A systematic review of the effect of retention methods in population-based cohort studies. BMC Public Health 2011;11:249.

35. Crutzen R, Viechtbauer W, Spigt M, et al. Differential attrition in health behaviour change trials: a systematic review and metaanalysis. Psychol Health 2015;30:122-34.

36. El Feky A, Gillies K, Gardner H, et al. A protocol for a systematic review of non-randomised evaluations of strategies to increase participant retention to randomised controlled trials. Syst Rev 2018;7:30.

37. Kim R, Hickman N, Gali K, et al. Maximizing retention with high risk participants in a clinical trial. Am J Health Promot 2014;28:268-74.

38. Thompson TP, Greaves CJ, Ayres R, et al. Factors associated with study attrition in a pilot randomised controlled trial to explore the role of exercise-assisted reduction to stop (ears) smoking in disadvantaged groups. Trials 2016;17:524.

39. Metse AP, Hizam NAN, Wiggers J, et al. Factors associated with retention in a smoking cessation trial for persons with a mental illness: a descriptive study. BMC Med Res Methodol 2018;18:177.

40. Belita E, Sidani S. Attrition in smoking cessation intervention studies: a systematic review. Can J Nurs Res 2015;47:21-40.

41. Stead LF, Koilpillai P, Fanshawe TR, et al. Combined pharmacotherapy and behavioural interventions for smoking cessation. Cochrane Database Syst Rev 2016;166.

42. Marcellus $L$. Are we missing anything? pursuing research on attrition. Can J Nurs Res 2004;36:82-98.
43. Curtin L, Brown RA, Sales SD. Determinants of attrition from cessation treatment in smokers with a history of major depressive disorder. Psychology of Addictive Behaviors 2000;14:134-42.

44. Prochaska JJ, Hall SE, Delucchi K, et al. Efficacy of initiating tobacco dependence treatment in inpatient psychiatry: a randomized controlled trial. Am J Public Health 2014;104:1557-65.

45. Brueton VC, Tierney J, Stenning S, et al. Strategies to improve retention in randomised trials. Cochrane Database Syst Rev 2013;12:1-126.

46. Coday M, Boutin-Foster C, Goldman Sher T, et al. Strategies for retaining study participants in behavioral intervention trials: retention experiences of the NIH behavior change Consortium. Ann Behav Med 2005;29 Suppl:55-65.

47. Moher D, Shamseer L, Clarke M, et al. Preferred reporting items for systematic review and meta-analysis protocols (PRISMA-P) 2015 statement. Syst Rev 2015;4:1.

48. Stockings E, Black N, Bartlem KM, et al. Outpatient interventions for smoking cessation and reduction for adults with a mental disorder. Cochrane Database Syst Rev 2019;105.

49. Civljak M, Bilic P, Milosevic M, et al. Interventions for smoking cessation in psychiatric settings. Cochrane Database Syst Rev 2018;(3).

50. Higgins J, Churchill R, Chandler J, et al. Cochrane Handbook for systematic reviews of interventions. version 5.2.0: the Cochrane collaboration. Available: www.training.cochrane.org/handbook [Accessed Jun 2017].

51. Adams M, Caffrey L, McKevitt C. Barriers and opportunities for enhancing patient recruitment and retention in clinical research: findings from an interview study in an NHS academic health science centre. Health Res Policy Syst 2015;13.

52. Liu Y, Pencheon E, Hunter RM, et al. Recruitment and retention strategies in mental health trials - a systematic review. PLoS One 2018;13:e0203127.

53. StataCorp. Stata statistical software: release 15. College Station, TX: StataCorp LLC, 2017.

54. Nyaga VN, Arbyn M, Aerts M. Metaprop: a Stata command to perform meta-analysis of binomial data. Arch Public Health 2014;72:39.

55. DerSimonian R, Laird N. Meta-Analysis in clinical trials. Control Clin Trials 1986;7:177-88.

56. Higgins JPT, Thompson SG, Deeks JJ. Measuring inconsistency in meta-analyses. BMJ 2003;327:557-60. 